EDITORIAL

\title{
Medicina de precisión y enfermedades cardiovasculares
}

\section{Precision medicine and cardiovascular diseases}

\author{
Luis Alberto Gómez Grosso ${ }^{\mathrm{a}, \mathrm{b}, \mathrm{c}, \mathrm{d}}$
}

\author{
a Grupo de Fisiología Molecular, Bogotá, Colombia \\ ${ }^{\mathrm{b}}$ Revista Biomédica, Bogotá, Colombia \\ c Instituto Nacional de Salud, Bogotá, Colombia \\ ' Departamento de Ciencias Fisiológicas. Facultad de Medicina, Universidad Nacional de Colombia, Bogotá, Colombia
}

Recibido el 30 de diciembre de 2015; aceptado el 15 de enero de 2016

Disponible en Internet el 8 de marzo de 2016

La medicina de precisión es un enfoque emergente de la medicina para la salud, la predicción, la prevención, el diagnóstico y el tratamiento de enfermedades. Este nuevo tipo de medicina basada en el conocimiento científico contemporáneo, tiene en cuenta la compleja relación biopsicosocial del individuo, amplía las perspectivas de interacción en red de informaciones médicas de diversos niveles, desde datos clínicos, de laboratorio, epidemiológicos y variabilidad individual de los genes, hasta estudios con modelos animales de enfermedades, informaciones genómicas, transcriptomas, factores de riesgo poblacionales, medio ambiente, estilo de vida, entre otras. Así mismo, es una respuesta a las necesidades y características específicas de los individuos ${ }^{1}$. Si bien los avances más significativos en cuanto a Medicina de precisión se han hecho en cáncer, la práctica cada vez tiende a extenderse a la mayoría de enfermedades y es particularmente necesaria en el manejo de las enfermedades cardiovasculares.

Es conocido ampliamente que estas últimas son un problema de salud pública y un reto global porque son las responsables de la mayor parte de la mortalidad en Colombia y en el mundo ${ }^{2}$. De las cuatro enfermedades crónicas no

Correos electrónicos: lgomez@ins.gov.co, lagomezg@unal.edu.co transmisibles principales incluyendo el cáncer, la diabetes y las enfermedades respiratorias crónicas, las enfermedades cardiovasculares fueron las causantes del 29,82\% (17 millones) de las muertes en $2011^{2}$. Se calcula que en 2030 morirán cerca de 23,6 millones de personas por enfermedades cardiovasculares y se pronostica que seguirán siendo la principal causa de muerte en todo el mundo ${ }^{2-5}$.

El impacto económico de las enfermedades cardiovasculares, es vasto. Por ejemplo, las pérdidas de producción en los países de ingresos bajos y medios (en dólares estadounidenses, US\$) pasarían de US\$ 3 billones en el 2010 a US\$ 8,6 billones en 2030. De igual forma, las estimaciones de los costos directos e indirectos por las enfermedades cardiovasculares en el mundo pasarían de US\$ 863 mil millones en 2010 a US\$ 1,04 billones en 2030 . Por tanto, las enfermedades cardiovasculares son una de las causas de pobreza que dificultan el desarrollo económico en todo el mundo y representan un enorme desafío global, en especial para los países en desarrollo como Colombia, en donde éstas corresponden al $28,7 \%$ de todas las defunciones ${ }^{5-7}$.

Por otra parte, pese a que las enfermedades cardiovasculares son más frecuentes en personas mayores de 60 años, el $25 \%$ de los fallecimientos por debajo de esta edad está relacionado con las enfermedades cardiovasculares, lo que sugiere que el aumento de su prevalencia también puede derivar, en parte, de un incremento en diferentes y 
múltiples factores de riesgo de naturaleza genética, epigenética, económica, socio-cultural, ambiental e incluso política, y que no necesariamente son excluyentes entre si ${ }^{5-7}$.

Por ejemplo, deficiencias en los sistemas de salud, en cuanto a su capacidad de realizar acciones de promoción y prevención, así como deficiencias en predicción, prevención, diagnóstico e intervenciones y tratamientos basados en la evidencia científica ${ }^{8,9}$, explican el aumento en los factores de riesgo de desarrollar enfermedad cardiovascular, tanto por factores conocidos como por factores de riesgo aun no conocidos suficientemente, incluyendo aquellos por alteraciones metabólicas, desórdenes alimentarios, obesidad abdominal, consumo de sustancias lícitas e ilícitas y cambios de estilo de vida saludable ${ }^{2-9}$, complejidad genómica, cambios y efectos epigenéticos, diversidad proteómica y metabolómica, así como por variedad de mecanismos de regulación celular y molecular, que hacen parte de la naturaleza humana y de sus ciclos de vida ${ }^{10}$.

En consecuencia, es urgente enfrentar la epidemia de las enfermedades cardiovasculares y debe ser una prioridad nacional e internacional en los sistemas de salud individuales y públicos en el mundo. De igual manera, conviene experimentar una transformación importante en torno a la calidad, al costo, al acceso, a las ideas innovadoras y a las aplicaciones prácticas para mejorar el panorama de las enfermedades cardiovasculares, con un nuevo modelo de medicina que permita acelerar la traducción de los conocimientos y descubrimientos biomédicos en nuevas herramientas de predicción, prevención, diagnóstico y terapias para el paciente y las poblaciones ${ }^{11,12}$.

Dado que las enfermedades cardiovasculares y la supervivencia a ellas son resultantes de la convergencia entre aspectos biológicos, sociales y culturales, la Medicina de precisión tiene un enorme potencial para avanzar en el conocimiento y manejo de la salud y de las enfermedades cardiovasculares humanas, así como para generar evidencia científica necesaria para aplicarla a la práctica clínica diaria y a la salud pública. La Medicina de precisión en el manejo de las enfermedades cardiovasculares, es considerada como la cardiología moderna del siglo XXI, ya que ofrece nuevas maneras de predecir, prevenir y detectar las enfermedades cardiovasculares en etapas tempranas y curables, facilita la identificación de pacientes susceptibles de responder a ciertos tratamientos y permite predecir la respuesta a estos ${ }^{13}$. Hoy más que nunca se requiere de una Medicina de precisión, que facilite intervenciones dirigidas hacia la predicción y prevención basadas en parte en el conocimiento de procesos moleculares y celulares específicos, que subyacen a la biología y a la patogénesis de las enfermedades cardiovasculares ${ }^{12-14}$. En un futuro cercano, será mejor predecir para prevenir tener que curar.

En concordancia con ello, la lucha contra las enfermedades cardiovasculares requiere una respuesta coordinada multisectorial. Las políticas de salud, las estrategias de investigación y los sistemas de salud en el mundo y en especial en países en desarrollo, se deben adaptar y fortalecer para enfrentar las enfermedades que tienden a desarrollarse más tarde en el ciclo de vida ${ }^{2,6,10}$. Así pues, se deben implementar estrategias dirigidas a las personas y a las poblaciones. Para este fin, la actuación integral en el ámbito nacional, regional y global es urgente y obligatoria para garantizar medidas eficaces en lo concerniente a la predicción, la prevención de la morbilidad, la discapacidad y la mitigación de la progresión de la enfermedad cardiovascular, es decir de una Medicina de precisión.

Existen intervenciones costo-efectivas basadas en la evidencia, para prevenir y controlar la amenaza de las enfermedades crónicas en los ámbitos mundial, regional, nacional y local. Factores de riesgo modificables como el tabaco, el consumo nocivo de alcohol, la dieta poco saludable, la actividad física insuficiente, la obesidad, así como la hipertensión, la hiperglucemia y la hipercolesterolemia son reconocidos como factores de riesgo principales, que contribuyen a la patogénesis subyacente de las enfermedades cardiovasculares $^{2-5}$. Estos riesgos han sido bien estudiados por medio de cambios en el estilo de vida y la dieta y se ha obtenido una disminución de los mismos. Se sabe que globalmente, los nueve factores de riesgo cardiovascular medibles y modificables (tabaquismo, hipertensión arterial, sobrepeso corporal, perímetro abdominal, hiperlipidemias por aumento de colesterol total, colesterol HDL, colesterol LDL y triglicéridos e hiperglucemia) representan el 70 al $90 \%$ del riesgo atribuible a la población, con estimaciones similares en la mayoría de las regiones del mundo, incluyendo a Colombia, India, China y otras partes de Asia ${ }^{6,8}$. Sin embargo, el efecto de las estrategias de intervención podría variar en función de factores biológicos, genéticos, socioeconómicos, ambientales y culturales particulares ${ }^{7,10}$.

La modificación profunda en el conocimiento científico requiere que hoy se le preste atención prioritaria a la Medicina de precisión en la atención de salud y la enfermedad cardiovascular. El conocimiento de los mecanismos celulares y moleculares de procesos fisiológicos normales que se pueden alterar en las enfermedades cardiovasculares, ha revelado una interacción compleja entre factores genéticos, epigenéticos, biológicos y ambientales (estilos de vida, aspectos sociales y culturales). En la actualidad, hay evidencia de que los cambios epigenéticos, definidos como cambios en la actividad de los genes que pueden ser heredables y que no dependen directamente de la secuencia del ADN, se modifican a medida que las células envejecen ${ }^{14}$. Además, el descubrimiento y la posibilidad de interferir las causas de enfermedades cardiovasculares específicas, para ayudar a mantener y mejorar la salud de las personas y de las poblaciones, plantean nuevos paradigmas para el desarrollo y la traducción de la información genómica y epigenómica en la práctica médica y en la integración de la medicina personalizada en la atención de salud.

Los análisis moleculares en el corazón revelan mecanismos de cardiopatía subyacentes y nuevas aproximaciones para la intervención terapéutica. Por ejemplo, en los últimos 10 a 15 años, la evolución de perfiles de expresión génica ha abierto nuevos espacios para el descubrimiento de factores importantes en la patogénesis de enfermedades cardiovasculares incluyendo la enfermedad de las arterias coronarias. Perfiles de ARN mensajero y de microARNs, se expresan diferencialmente en los pacientes con placas coronarias, ruptura de placa aguda y en respuesta a los tratamientos para los síndromes coronarios agudos ${ }^{15}$. Uno de los propósitos es integrar la información de los perfiles de ARN mensajero de los microARN en cada etapa de la progresión de un paciente a través de la historia natural de la enfermedad cardiovascular, incluyendo la evaluación de factores de riesgo, 
predicción y detección de la enfermedad arterial coronaria y el síndrome coronario agudo, y finalmente, en la respuesta a los tratamientos para la enfermedad de las arterias coronarias y sus secuelas, incluyendo la insuficiencia cardíaca congestiva. Estos enfoques también tienen implicaciones significativas para la detección no invasiva y el seguimiento de enfermedades cardiovasculares, la estratificación terapéutica y la identificación de aciertos y fallas a las terapias dirigidas $^{16}$.

Por su parte, la secuenciación masiva en paralelo puede empoderar a los cardiólogos a tomar decisiones de tratamiento informadas por la composición molecular de sus pacientes, a través del desarrollo e implementación de una plataforma de perfiles moleculares robusta para su uso en el tratamiento en tiempo real en los grandes centros de cardiología y su utilidad en la adecuación de los pacientes a los ensayos clínicos y de investigación con mayor posibilidad de éxito ${ }^{11}$.

Numerosos factores convergen para promover la adopción de prácticas de Medicina de precisión en todo el mundo, tales como mayor poder estadístico de la investigación molecular acumulada, costo decreciente de almacenamiento de datos, junto con el auge de las tecnologías móviles y uso generalizado de las historias médicas en medios electrónicos. Además, el porcentaje de terapias dirigidas a dianas moleculares ha ido en constante aumento en el número total de medicamentos aprobados y está demostrando ser el camino a seguir ${ }^{17-20}$. Este editorial hace un llamado a los líderes en las comunidades científicas y médicas, así como a profesionales de la salud, para empezar a generar una nueva visión y usar la experiencia profesional con el objetivo de acelerar los descubrimientos biomédicos y traducirlos en herramientas de predicción, prevención, diagnóstico y tratamiento precisos de enfermedades críticas como las cardiovasculares.

No obstante, la puesta en práctica de la Medicina de precisión en el manejo de las enfermedades cardiovasculares requiere enfrentar algunas barreras para su adopción, incluyendo el alto costo de las pruebas, la escasez de ensayos clínicos aleatorizados que demuestren la eficacia, la falta de plataformas de ensayo adecuadas y aprobadas por las agencias de control y vigilancia de medicamentos, la implementación de la Farmacogenética, la Farmacogenómica, la Genómica, las tecnologías de la salud digital, la evaluación de los pacientes y de las poblaciones que se presentan con el genoma secuenciado, la traducción de estos análisis a la clínica, el uso de nuevas tecnologías, que han surgido para analizar las alteraciones moleculares en el ADN, cambios en ARNs largos no codificantes, en los miARNs en la circulación sanguínea de pacientes con enfermedad coronaria, la integración y utilización de los datos clínicos y genómicos, los ensayos clínicos, el cubrimiento de la gran cantidad de datos (big data) en biomedicina, el desarrollo de una plataforma para la investigación científica-tecnológica y la innovación, la simplificación de lo aparentemente complejo, el escaso conocimiento e interés entre los médicos especialistas, los médicos generales, otros profesionales de la salud, los estudiantes, los pacientes, los tomadores de decisiones y el público en general ${ }^{2-10,14}$.

Además, el potencial de la Medicina de precisión requiere un ecosistema global que pueda acumular datos sobre cientos de millones de pacientes en el mundo, con el fin de identificar las mejores terapias para enfermedades cardiovasculares comunes y raras, desarrollar modelos de negocio audaces y tecnologías innovadoras que fomenten la calidad y el intercambio de datos a través de las partes interesadas, y conformar redes de prevención, de diagnóstico y de investigación de enfermedades cardiovasculares para el intercambio de datos, la reducción de los costos y la aceleración del aprendizaje ${ }^{16}$.

La aplicación e implementación de una Medicina de precisión y de un plan de acción para la predicción, la prevención y el control de algunas enfermedades cardiovasculares comunes, la estrategia mundial sobre régimen alimentario y para reducir el uso nocivo del alcohol, además de la actividad física son iniciativas globales y vitales ${ }^{2,12}$. El diseño y la ejecución de modelos de atención más costoefectivos, como se proyecta con una Medicina de precisión, que dependen de los profesionales de la salud capacitados, pueden generar un mayor impacto en las personas en riesgo y con enfermedad cardiovascular.

En este contexto, se proponen recomendaciones a escala mundial, que incluyen una Medicina de precisión en cardiología orientada a la predicción, la promoción, la prevención, la detección temprana, las terapias dirigidas y selectivas, así como el desarrollo de programas globales y locales de vigilancia, monitorización y evaluación aplicadas a toda la población en riesgo. Además, se requiere el desarrollo de acciones rápidas para incrementar y acelerar la generación e integración del conocimiento sobre los determinantes biológicos, la etiología y la patogénesis de las enfermedades cardiovasculares.

Por otro lado, es importante continuar trabajando en investigación científica biomédica y clínica para la incorporación de nuevos avances en Medicina molecular en pro del cuidado de los pacientes y de las poblaciones ${ }^{12}$. En este sentido, para que los nuevos descubrimientos sean beneficiosos para los pacientes y la población en riesgo de desarrollar enfermedades cardiovasculares, se precisan diferentes pasos integrados en una cadena de valor. Por ejemplo, se parte de las necesidades y de los vacíos en el conocimiento para afrontar problemas y necesidades de salud global no resueltos, se formulan y se desarrollan investigaciones iniciales; luego, el conocimiento científico inicial puede conducir al desarrollo y la evaluación de potenciales aplicaciones, bienes y servicios en salud, seguido por la validación y la prescripción de recomendaciones basadas en la evidencia, para la práctica clínica y para la toma de decisiones en salud.

Varios países en el mundo y recientemente con el apoyo de presidente de Estados Unidos, Barak Obama, realizan esfuerzos de inversión, técnicos y administrativos para que la Medicina de precisión sea la norma y no la excepción. Esta iniciativa es un esfuerzo nuevo y audaz para revolucionar la forma en cómo mejorar la salud y combatir la enfermedad. La Medicina de precisión se presenta como un nuevo modelo para acelerar la traducción de los conocimientos y descubrimientos biomédicos en nuevas herramientas predictivas, de prevención, diagnóstico, terapias e intervenciones para los pacientes y las poblaciones ${ }^{1}$.

Se propone, así mismo, que la Medicina de precisión será la Medicina del siglo Xxı, porque puede ofrecer nuevas maneras de predecir, prevenir y detectar las enfermedades en etapas tempranas y curables y también facilitar la 
identificación de los pacientes susceptibles de responder a ciertos tratamientos y predecir la respuesta a estos.

En definitiva, la aplicación de una medicina de precisión transformará la manera en que podemos tratar las enfermedades crónicas no transmisibles, como las cardiovasculares, hecho que permitirá a los médicos practicar la medicina predictiva y preventiva, así como seleccionar herramientas diagnósticas y tratamientos que mejoren las posibilidades de supervivencia y reducción de la exposición a efectos adversos. La Medicina de precisión tiene un enorme potencial en el manejo de la salud y de las enfermedades cardiovasculares, al igual que para generar la evidencia científica necesaria para aplicarla a la práctica clínica diaria y a la salud pública.

\section{Bibliografía}

1. Collins FS, Varmus $\mathrm{H}$. A new initiative on precision medicine. $\mathrm{N}$ Engl J Med. 2015;372:793-5.

2. Gómez LA. Las enfermedades cardiovasculares: un problema de salud pública y un reto global. Biomédica. 2011;31:469-73.

3. World Health Organization. Global status report on non communicable diseases 2011. Disponible en: http://www.who.int/ $\mathrm{nmh} /$ publications/ncd_report2010/es/. [Acceso 25 Nov 2015].

4. World Health Organization. New HWO report. April 27, 2011. Disponible en: http://www.who.int/mediacentre/news/ releases/2011/ncds_20110427/en/. [Acceso 25 Nov 2015].

5. Roger VL, Go AS, Lloyd-Jones DM, Adams RJ, Berry JD, Brown TM, et al. Heart disease and stroke statistics-2011 update: a report from the American Heart Association. Circulation. 2011;123:e18-209.

6. Butler D. UN targets top killers. International summit considers how to stem the rise in non-communicable diseases. Nature. 2011;477:260-1.

7. Abegunde DO, Mathers CD, Adam T, Ortegon M, Strong K. The burden and costs of chronic diseases in low-income and middleincome countries. Lancet. 2007;370:1929-38.

8. Brownson RC, Haire-Joshu D, Luke DA. Shaping the context of health: a review of environmental and policy approaches in the prevention of chronic diseases. Annu Rev Public Health. 2006;27:341-70.

9. Moncayo A. Nuevas dimensiones de la salud pública. En: Malagon-Londoño, Moncayo-Medina. Salud Pública.
Perspectivas. Cap. 2, $2^{\text {a }}$ ed. Bogotá, D.C. Editorial Médica Panamericana, Internacional Ltda. 2011. p. 26-44.

10. Gómez LA. Avances científicos y tecnológicos. Genoma humano y salud pública. Cap. 37. En: Malagon-Londoño, MoncayoMedina. Salud Pública. Perspectivas. $2^{a}$ ed. Bogotá, D.C. Editorial Médica Panamericana, Internacional Ltda. 2011. p. $615-30$.

11. Kingsmore SF, Petrikin J, Willig LK, Guest E. Emergency medical genomes: a breakthrough application of precision medicine. Genome Medicine. 2015;7:82-8.

12. Hui Y, Zhang VW. Precision Medicine for Continuing Phenotype Expansion of Human Genetic Diseases. BioMed Research International Volume 2015, Article ID 745043, 4 pages. http://dx.doi.org/10.1155/2015/745043

13. Dankwa-Mullan I, Bull J, Sy F. Precision Medicine and health disparities: Advancing the science of individualizing Patient Care. Am J Pub Health. 2015;105 Suppl 3:S368.

14. Gomez LA. XII Encuentro científico "Desarrollo, innovación y salud". Biomédica. 2011;31:11-2

15. Kovacic JC, Fuster V. From treating complex coronary artery disease to promoting cardiovascular health: therapeutic transitions and challenges, 2010-2020. Clin Pharmacol Ther. 2011;90:509-18.

16. Lopez-Campos GH, Lopez-Alonso V, Martin-Sanchez F. Is Precision Medicine different from Personalized Medicine? A Biomedical informatics perspective. Integrating Information Technology and Management for Quality of Care J. Mantas et al. (Eds.) IOS Press, 2014. doi:10.3233/978-1-61499423-7-20.

17. Dyer MJS, Vogler M, Samuel J, Jayne S, Wagner S, Pritchard C, Macip S. Precision medicines for B-cell leukaemias and lymphomas; progress and potential pitfalls. Brit J Haematol. 2013;160:725-33.

18. Duffy MJ, John Crown J. Precision treatment for cancer: Role of prognostic and predictive markers. Crit Rev Clin Lab Sci. 2014;51:30-45.

19. Servant N, Roméjon J, Gestraud P, La Rosa Ph, Lucotte G, Lair S, et al. Bioinformatics for precision medicine in oncology: principles and application to the SHIVA clinical trial. Frontiers in Genetics | Systems Biology. 2014;5:1-16.

20. Choi YY, Noh SH, Cheong JH. Evolution of gastric cancer treatment: from the golden age of surgery to an era of precision medicine. Yonsei Med J. 2015;56:1177-85. 